\title{
EVALUACIÓN DE LA PERCEPCIÓN DE UN PROGRAMA TUTORIAL DIRIGIDO A ESTUDIANTES DE PROVINCIA EN UNA UNIVERSIDAD DE LIMA
}

\section{PERCEPTION ASSESSMENT OF A TUTORIAL PROGRAM AIMED AT INLAND STUDENTS AT A LIMA UNIVERSITY}

\author{
Nohemí Estefani Marcelo Torres* \\ Universidad Peruana de Ciencias Aplicadas, Perú
}

\section{RESUMEN}

El acompañamiento tutorial, en los últimos años, ha venido cobrando relevancia debido a la implicancia que tiene en el desempeño socioemocional y académico de los estudiantes, es por ello que muchas universidades diseñan planes institucionales que permiten a sus estudiantes fortalecer habilidades y capacidades. En ese sentido, el presente estudio pretende conocer cuáles son las percepciones que tiene un grupo de 30 estudiantes, entre 15 y 18 años, provenientes del interior del país, respecto a las actividades desarrolladas en el programa tutorial del semestre 2014-1. Se empleó un diseño cualitativo de tipo fenomenológico, y para la recolección de datos se emplearon grupos focales y técnicas proyectivas. Los resultados indican que el programa tutorial es concebido por los estudiantes como un espacio de apoyo, escucha que favorece al proceso de adaptación a la vida universitaria, aunque, también, señalan que éste debe ajustarse de acuerdo a las constantes necesidades del grupo.

Palabras clave: tutoría, adaptación, acompañamiento tutorial.

\section{ABSTRACT}

Tutorial support has become more relevant in recent years because of its implications on the socio-emotional and academic performance of students. Consequently, many universities are implementing institutional plans designed to enable students to strengthen their skills and abilities. In that sense, the study seeks to examine the perception of a group of 30 inland students, between the ages of 15 and 18, with respect to the activities developed during the 2014-1 tutorial program. A qualitative phenomenological design was used, as well as focus groups and projective techniques for the data collection process. The results indicate that students view the tutorial program as a space for support and attention, which contributes to the college-life adjustment process; however, they indicate that it should be adapted to the group's ever-changing needs.

Keywords: tutoring, adjustment, tutorial support. 


\section{INTRODUCCIÓN}

Actualmente, la tutoría universitaria es considerada como una herramienta de gran importancia en la formación de los estudiantes, ya que implica actividades orientadas a formar a los alumnos a nivel personal, social y profesional (Ferrer, 2003). Para Crockett, la tutoría es un espacio donde se provee una orientación sistemática a lo largo de todo el trayecto académico; asumiendo el alumno (y el docente) la responsabilidad en el proceso de clarificación de objetivos de carrera y vida, toma de decisiones y resolución de conflictos (como se citó en Upcraft, Gardner y Barefoot, 2005).

Esto supone un proceso de cambios en las instituciones de educación superior, a nivel académico y organizativo, ya que las universidades deberán formular planes y estrategias que involucren una serie de recursos económicos, materiales y personales que convierta al alumno en uno de los pilares más importantes del cambio, lo cual significa una mejora en la experiencia educativa, ya que se parte de la necesidad del alumno (Romo, 2011).

Esta relevancia surge de los diversos cambios que viene atravesando nuestra sociedad, donde la educación, específicamente la Universidad, debe responder a las diversas demandas sociales y esto se realiza a través de la formación integral de los estudiantes, contribuyendo con la transmisión de la cultura, ciencia e investigación (Álvarez González, 2004; Amor, 2012; Bausela, 2006; Bisquerra, 1998; Echeverría, 1997; García Nieto, 2008; Lázaro, 1997; Pantoja y Campoy, 2009; Repetto, 2002; Rodríguez Espinar, 2004; Rodríguez Moreno, 2002; Zabalza, 2003).

Al ser la acción tutorial un proceso de acompañamiento continuo, está presente a lo largo de toda la vida académica y la etapa universitaria no es la excepción. En consecuencia, diversos estudios demuestran la necesidad e importancia de establecer lineamientos institucionales que permitan la orientación y asesoramiento al alumnado (Amor, 2012; Apodaca y Lobato, 1997; Colvin, 2007; González López y Martín Izard, 2004; Pino y Ricoy, 2006; Sanz Oro, 2001; Watts y Van Esbroeck, 1998).

En concordancia, algunas investigaciones demuestran que los estudiantes universitarios están más conscientes y comprometidos con su formación profesional, por lo cual esperan que la universidad les permita vivir experiencias donde puedan desarrollar nuevas capacidades, adquirir madurez, es decir, obtener una preparación no sólo profesional sino también personal que les permitan afrontar diversas situaciones que puedan presentárseles en la vida (Álvarez Pérez, Alegre de la Rosa y LópezAguilar, 2012; Bishop, 1990; Castellano, 1995; Castellano y Sanz, 1990; Coriat, 2002; Coriat y Sanz, 2005; Michavila y García, 2003; Saíz y Román, 2011; Stone y Archer, 1990).

Por lo anterior expuesto, es importante definir un modelo tutorial que esté alineado con la misión y visión de la universidad y que responda a las demandas de los estudiantes, la institución y la sociedad (Porta, como se citó en Rodríguez Espinar, 2004); estos modelos van a definir las funciones del docente y los tres grandes ámbitos a trabajar en el sistema tutorial:

- Tutoría vocacional (tránsito de la secundaria-universidad)

Este servicio constituye un entrenamiento en la toma de decisiones, válidas y coherentes, que permitan la identificación del perfil que poseen y cómo éste se ajusta a los requerimientos de la 
carrera. Si bien es cierto, esta actividad debería desarrollarse en el período de educación básica regular, en la práctica no siempre sucede así. Esto implica que los estudiantes lleguen a la universidad con una sería confusión respecto a su quehacer profesional. Por ello, es importante que se desarrollen actividades que permitan a los estudiantes aclarar y guiar su vocación, esto se realiza a través del diseño y aplicación de programas breves de orientación académica y vocacional.

- Tutoría académica-universitaria (docente y orientadora)

Elobjetivo principal es brindar apoyo académico, personal y profesional a los estudiantes a los largo de la carrera; en conclusión, busca contribuir con el desarrollo integral de las personas que transitan por la universidad, fortaleciendo los aspectos positivos $\mathrm{y}$ trabajando en los puntos débiles, manteniendo una visión de enseñanza-aprendizaje centrada en el desarrollo de conocimientos, habilidades y actitudes (competencias), con el fin de la consecución eficaz de objetivos.

Por esta razón, es importante que el trabajo se centre en una atención personalizada y profunda, formando grupos pequeños que permitan el establecimiento de vínculos producto de un ambiente de confianza e intimidad.

- Tutoría profesional-laboral (inserción laboral)

El objetivo es fortalecer la capacitación profesional de los alumnos e insertarlos al mercado laboral.

El establecimiento de un modelo, en la práctica tutorial, permite a las instituciones desvincular la concepción inicial, sujeta a la orientación puramente académica, para asignarle un nuevo significado al acompañamiento del estudiante brindado a varios niveles en donde se formule un espacio para orientar los aprendizajes y las futuras prácticas (Michavila y García, 2003; Rué, 2004).

Por ello, tal como lo señalan diversos autores, un modelo integral de tutoría es el que aborda aspectos académicos, profesionales y personales del estudiante, ya que permite el fortalecimiento de diversas facetas del alumno: intelectual, afectiva y profesional (Álvarez Pérez, et al., 2012; García y Gálvez, 1996; Marín y Rodríguez Espinar, 2001).

Tal como lo señala Castro (2012), este modelo requiere de una gran dedicación y capacitación del profesorado (o profesional a cargo de las tutorías), ya que se pretende que desempeñe diversos roles complejos para que pueda realizar un acompañamiento óptimo, lo cual genera, a su vez, la necesidad de contar con una red de servicios de apoyo.

Asimismo, diversos estudios señalan variados beneficios, atribuidos a este modelo: 1) Favorece el desarrollo integral de los estudiantes, en la medida que abarca dimensiones fundamentales en su formación (intelectual, personal, afectiva y social). 2) Permite una atención individualizada. 3) Brinda información al estudiante, que le posibilita integrarse en la comunidad universitaria y su proceso formativo (administrativos, de currículo, actividades académicas formativas y recreativas-culturales, actividades de orientación, desarrollos de servicios, etc.). 4) Mejora los contextos de enseñanzay aprendizaje, ya que se configuran espacios acogedores y desafiantes (intelectualmente y afectivamente). 5) Promueve la participación de los estudiantes en la institución (Álvarez Pérez y Jiménez, 2003; Álvarez Pérez, et al., 2012; Álvarez Rojo, García, Gil y Romero, 2000; Castro, 2012; Haya, Calvo y Rodríguez-Hoyos, 2013; Lobato y Echevarría, 2004; Rodríguez Espinar, 2004). 
Por lo anteriormente expuesto, es importante contar con profesionales que tengan comprobadas competencias académicas, emocionales, psicológicas y sociales para el trabajo con estudiantes en proceso de formación.

Castro (2012) y Gallego (como se citó en Gairín, Feixas, Guillamón y Quinquer, 2004) refieren que el tutor debe presentar ciertas características que lo definan para las funciones que va a desempeñar:

Se espera que estos docentes desarrollen competencias a cuatro niveles:

1.-Cognitivo:Conocerlaspolíticasdelainstitución, los proyectos de la universidad, identificarse con la misión y visión de la organización, reconocer los procesos administrativos que atañen a los estudiantes, los procesos de desarrollo académico, social, emocional y psicológico por los cuales atraviesan los estudiantes.
2.- Técnico: Conocer y manejar técnicas empleadas en la labor tutorial (entrevista, observación, dinámicas de grupos, estrategias de seguimiento y monitoreo, etc.). Poseer habilidades de comunicación efectiva, resolución de conflictos, pensamiento reflexivo, negociación, entre otros. Mantener contacto con las diversas áreas de Orientación y bienestar al educando, con la finalidad de realizar derivaciones oportunas.

3.- Afectivo: Autoconocimiento, autoaceptación, capacidad de expresión de necesidades, tolerancia a la frustración y el estrés, respeto por la individualidad y la diversidad académica, cultural y socio-económica, demostrar empatía, consideración por las limitaciones propias y ajenas, capacidad de comunicación asertiva, manejo de códigos éticos y deontológicos, valorar la dignidad de la persona.

4.- Social: Saber reír en momentos apropiados, mantener la calma en situaciones difíciles

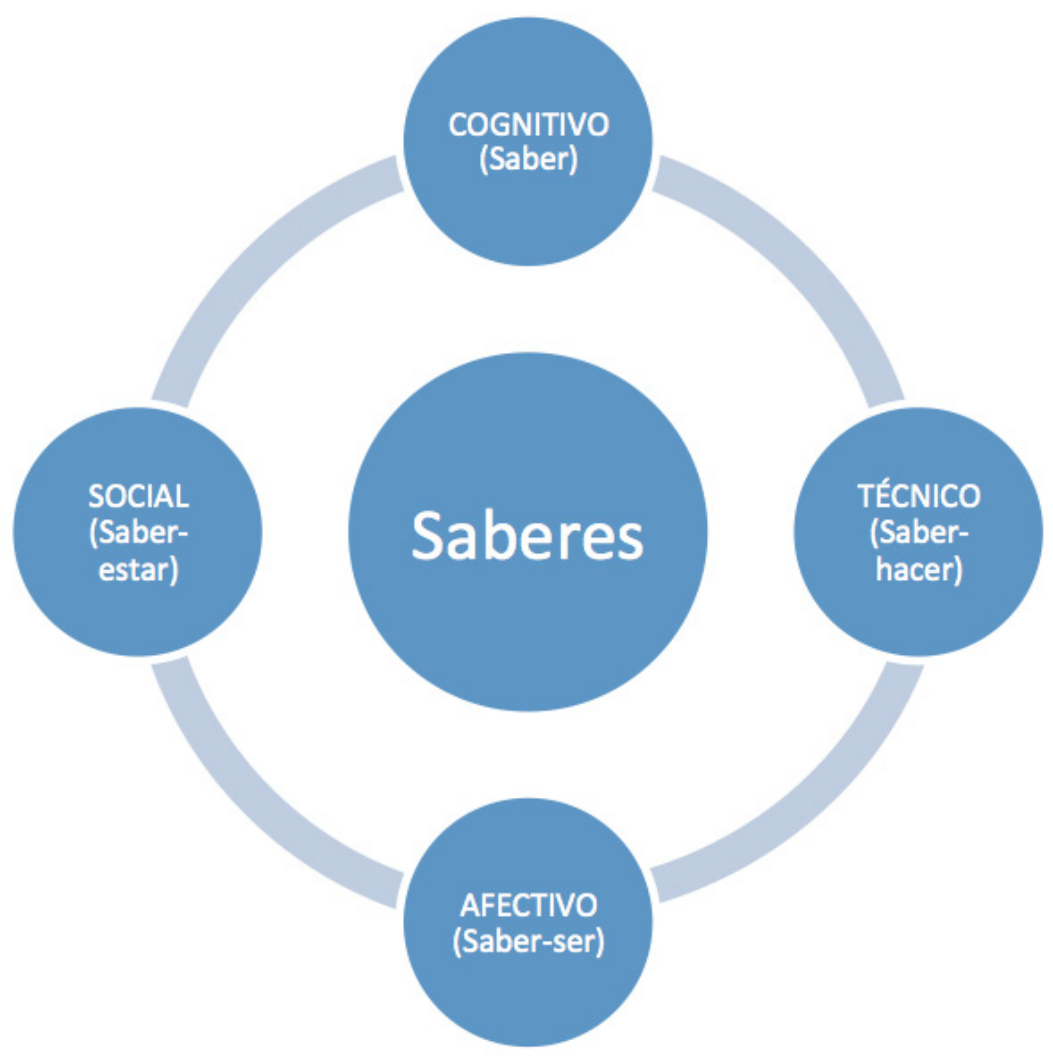

Imagen 1. Competencias docente-tutor 
y reflejar un estado de ánimo apropiado a la circunstancia.

En definitiva, el presente estudio busca conocer, desde la óptica de los protagonistas, en este caso el alumnado, las percepciones que tienen acerca del programa tutorial. Por ello, este trabajo, además de recoger los aportes de los estudiantes, busca incorporarlos dentro del ámbito del diseño y la implementación de actividades que respondan de manera imparcial a sus necesidades.

\section{Objetivos}

El presente estudio pretendió “Evaluar las percepciones del programa tutorial en una universidad privada de Lima", teniendo como objetivos específicos:

- Comprender el proceso de adaptación a la vida universitaria.

- Interpretar las fortalezas y debilidades del programa de tutoría.

- Identificar las sugerencias, acerca de la metodología, que se deben utilizar en el programa de tutoría.

\section{METODOLOGÍA}

\section{Diseño}

La metodología empleada fue cualitativa de tipo exploratoria e interpretativa, pues permite conocer de forma individual y profunda la percepción de los estudiantes. Con respecto al diseño, es fenomenológico (Creswell, Hanson, Clark \& Morales, 2007).

\section{Participantes}

El grupo evaluado estuvo compuesto por jóvenes de provincia, entre los 15 y 18 años de edad, pertenecientes a las diversas carreras que tiene la universidad.

En total se evaluó a 30 estudiantes, pertenecientes a dos campus de la universidad, con los cuales se organizó tres grupos de 10 alumnos cada uno. Del total de pupilos, 20 continúan en el programa tutorial y 10 abandonaron el servicio.

\section{Técnicas de recolección de datos}

Para la recolección de datos se aplicó la técnica de grupos focales, apoyada por el empleo de una entrevista semiestructurada. Esta actividad fue dirigida y registrada por el entrevistador con el objetivo de obtener información (opiniones y sentimientos) que responden al objetivo de estudio. Adicionalmente, se utilizaron técnicas proyectivas de animación y collage, con el fin de identificar emociones y percepciones encubiertas.

\section{PROCEDIMIENTO}

El estudio se realizó en las aulas de la universidad y fue conducido por una especialista con amplia experiencia en este tipo de investigación.

Asimismo, se respetaron los principios éticos de confidencialidad, anonimato y máxima reserva de los datos, y se les señaló a los participantes que los resultados obtenidos sólo serían utilizados para el presente estudio con el propósito de plantear mejoras. En ese sentido, se les hizo firmar un consentimiento informado para garantizar su participación voluntaria en esta investigación.

Se realizaron las transcripciones y se procedió al análisis de contenido de sus comentarios y la observación de imágenes que se efectuaron en el collage.

\section{RESULTADOS Y DISCUSIÓN}

Los resultados se muestran en función a tres aspectos específicos, sobre los cuales se indagó: adaptación a la universidad, debilidades $\mathrm{y}$ fortalezas del programa $\mathrm{y}$, finalmente, sugerencias planteadas. 


\section{Adaptación a la universidad}

Este aspecto permitió conocer cuál fue el proceso que tuvieron que atravesar los estudiantes para insertarse en la vida universitaria y si las actividades planteadas, en el programa tutorial, permitieron fortalecer ciertas capacidades para el afronte de esta situación.

En ese sentido, el trasladarse de provincia a la capital significó un cambio drástico, ya que tuvieron que asumir las diferencias culturales, aprender a ser independientes, responsables y autónomos en sus decisiones y acciones. Experimentando un sentimiento de desarraigo y añoranza, sumado a ello tuvieron que establecer nuevos vínculos y relaciones amicales con diversas personas, lo cual les generaba cierto grado de ansiedad.

"Bueno, para mí fue un cambio muy drástico, de saber que tienes que encargarte del dinero, de nosotros mismos, estudios, exámenes, muchas veces tienes que estar pendiente, no descuidar porque malogras todo."

(Estudiante que permanece en el Programa Tutorial - Sede Chorrillos)

"Bueno, la verdad alejarme de mi familia ha sido algo muy complicado porque la mayoría de las veces siempre he vivido con mi familia, también acerca del método de estudio que tiene la Universidad respecto a eso, si me ha chocado bastante."

(Estudiante que permanece en el Programa Tutorial - Sede Chorrillos)

"Una persona, o sea cuando llegué me parecía todo extraño porque conocer a personas nuevas, la expresión es algo asombroso encontrarte con personas que piensan diferente a ti o que muchas veces coinciden."
(Estudiante que abandonó el Programa Tutorial - Sede Chorrillos)

“...Conocer a personas nuevas no fue tan fácil para mí, como yo hubiera esperado, cada persona tenía tanta capacidad como la mía o tal vez más,..."

(Estudiante que abandonó el Programa Tutorial - Sede Chorrillos)

Estos sentimientos, se pusieron en evidencia en los dibujos que realizaron los estudiantes:

"Yo dibujé todo mi cuerpo como si se estuviese mutilando, que en ese momento se me vinieron muchos sentimientos positivos y negativos."

(Estudiante que abandonó el Programa Tutorial - Sede Chorrillos)

"Yo dibujé una persona con interrogantes."

(Estudiante que abandonó el Programa Tutorial - Sede Chorrillos)

"Un ambiente con personas nuevas, un ambiente nuevo, con personas extrañas o sea como recién empiezas, no las conoces bien."

(Estudiante que abandonó el Programa Tutorial - Sede Chorrillos)

Es importante señalar que este grupo se siente más integrado con los estudiantes que también provienen del interior del país, gran parte de los estudiantes se siente distanciado de los alumnos de Lima.

Tal comolo señalan Álvarez Pérezy González Alfonso (2007), las dificultades de adaptación personal y social en los alumnos ingresantes (en este caso inmigrantes) son producto de la falta de habilidades sociales, soledad, problemas de integración, separación de familiares, indefensión, escasa autonomía, etc., lo cual pone de manifiesto la falta de equilibrio emocional. 


\section{Percepción de la universidad}

Respecto a las fortalezas que reconocen en la universidad, tanto los estudiantes que continúan en el programa tutorial como los que dejaron el servicio, destacan la infraestructura moderna, la tecnología, la oportunidad que les brinda para aprender cosas nuevas, contar con un plan de profesionales calificados, buena organización, oportunidades para la carrera y facilidad para la formación profesional en el extranjero.

"Cuando estoy aquí siento que estoy aprendiendo nuevas cosas, o conociendo."

(Estudiante que permanece en el Programa Tutorial - Sede Chorrillos)

"La universidad te ayuda a madurar y todo eso, pero te presiona, te consume."

(Estudiante que permanece en el Programa Tutorial - Sede Chorrillos)

"Yo pensé en el descerebrado, por que como dicen te vuelves loco en la Universidad, muchas cosas de estudio, muchas cosas que pasas que implica la Universidad que tienes que madurar a una velocidad, tienes que ser responsable cada vez más."

(Estudiante que Permanece en el Programa Tutorial - Sede Chorrillos)

"Un ambiente con personas nuevas, un ambiente nuevo, con personas extrañas y o sea como recién empiezas no las conoces bien pero que poco a poco ya los vas a ir conociendo y probablemente puedan ser más amigos."

(Estudiante que abandonó el Programa Tutorial - Sede Chorrillos)

"Tiene buena infraestructura, moderna, cuenta con buenos profesionales."
(Estudiante que abandonó el Programa Tutorial - Sede Surco)

"Es bastante organizado, tiene oportunidades para la carrera y facilidades de formación con respecto a la carrera."

(Estudiante que abandonó el Programa Tutorial - Sede Surco)

Sin embargo, algunos estudiantes identifican, también, algunas debilidades en relación a la calidad de enseñanza y no la consideran uniforme, existen profesores que no son percibidos como "buenos". Se quejan de algunos servicios, como que los cubículos son muy pequeños, mucha cantidad de alumnado, la biblioteca no tiene el espacio necesario como para albergar a los estudiantes.

"Calidad de profesores, hay profesores buenos como también algunos profesores que son vagos."

(Estudiante que abandonó el Programa Tutorial - Sede Surco)

\section{Comunicación familiar}

Gran parte de los estudiantes tiene una comunicación fluida con su familia, mantienen un vínculo de confianza y comentan con sus padres las dificultades que se les presentan y las actividades que realizan; empero, algunos de los alumnos refieren que no manifiestan sus preocupaciones a sus progenitores para evitar preocuparlos o que se molesten.

Un grupo menor de los estudiantes que abandonaron la tutoría, mantiene una comunicación un poco más distante una vez al mes, se muestran más independientes; por lo general, no suelen dialogar mucho con sus padres de las contrariedades y los aprietos que pueden haber pasado. 
“Normalmente, mi Mamá me llama siempre.”

(Estudiante que permanece en el

Programa Tutorial - Sede Chorrillos)

"Sí, siempre me he comunicado con mi Papá, todos los días, le llamo, me llaman. Creo que dejar de lado los problemas y para saber cómo está y un momento de relajo, no para estar ahí triste."

(Estudiante que permanece en el Programa Tutorial - Sede Chorrillos)

"Una vez al mes, depende de las circunstancias."

(Estudiante que abandonó el Programa Tutorial - Sede Chorrillos)

"De vez en cuando, nomás, 1 ó 2 veces por mes."

(Estudiante que abandonó el Programa Tutorial - Sede Surco)

"Yo sí tengo RPM y me comunico 5 ó 6 veces a la semana, más hablo con mi Papá que con mi Mamá, porque cuando hablo con mi Mamá, siento que va a llorar. Ella es bastante sentimental y no me gusta entonces escuchar eso y ya trato de cortar... pero más hablo con $\mathrm{mi}$ Papá."

(Estudiante que abandonó el Programa Tutorial - Sede Surco)

Las características de los alumnos van a determinar las necesidades emocionales que deben ser cubiertas. Mientras el alumno muestre mayor autonomía, capacidad de diálogo y establecimiento de vínculo, el proceso de adaptación será progresivo y positivo (Castro, 2012).

La familia juega un rol fundamental en el proceso de adaptación de los estudiantes, ya que les permite conectarse con su cultura, tradiciones, costumbres, etc. El alumno, al insertarse en un nuevo contexto, debe encarar este hecho y asumir una nueva postura que le posibilite sentirse parte de un nuevo grupo, con nuevo códigos de conducta, nuevas costumbres e, incluso, un sistema de valores diferenciado. Por ello, mantener una comunicación constante con sus familiares, le permite atravesar este proceso de manera sistemática (Díaz, Tarragona y Trejos, 2007).

\section{Debilidades y fortalezas del programa tutorial}

\section{Situación actual}

La mayoría de los participantes, piensa que estudiar en la universidad es una fuente de satisfacción personal y una manera de alcanzar el éxito, lo cual se logra a base de mucho esfuerzo y sacrificio. Terminar los estudios, implica la posibilidad de la mejora en las condiciones económicas del estudiante y de su familia.

Los alumnos reflejaron su sentir a través de los dibujos que realizaron, además, explicaron lo que representaban:

"La Flecha subiendo: Superación, nos ayuda a mejorar intelectualmente y psicológicamente como personas."

(Estudiante que permanece en el Programa Tutorial - Sede Chorrillos)

“La lámina verde que es un triángulo de personas: Que eres tú el que decide donde estás, nos dice que depende de nosotros."

(Estudiante que permanece en el Programa Tutorial - Sede Chorrillos)

"La Antena satelital: Siempre estamos acompañados de la tecnología que nos dan."

(Estudiante que permanece en el Programa Tutorial - Sede Chorrillos) 
"Una boxeadora: Los alumnos representan los boxeadores, o sea todos sabemos de dónde venimos, por así decirlo, de qué hueco venimos y hasta donde debemos llegar."

(Estudiante que abandonó el Programa Tutorial - Sede Surco)

"Sueños de TUTÚ: que representa los sueños que podemos alcanzar."

(Estudiante que abandonó el Programa Tutorial - Sede Surco)

"Bicicleta: significa seguir adelante con nuestros sueños es audaz, se nota la fuerza, el esfuerzo y siempre para arriba."

(Estudiante que abandonó el Programa Tutorial - Sede Surco).

"Edificio: La infraestructura, la universidad moderna como que te da esas ganas de seguir, o sea, si tú vienes de otras Universidades no todos tienen estas cosas, las tecnologías que tenemos aquí no y que motivan bueno a seguir estudiando que no se duerma, el mismo ambiente."

(Estudiante que abandonó el Programa Tutorial - Sede Surco)

"Señor con casco: Nuestra meta es ser ingeniero."

(Estudiante que abandonó el Programa Tutorial - Sede Surco)

"Una chica sacando la lengua, es como decir a mal tiempo buena cara, de una manera te ayuda al desarrollo a darle una oportunidad."

(Estudiante que abandonó el Programa Tutorial - Sede Chorrillos)

\section{Fortalezas del programa}

Los alumnos que tienden a tener percepciones positivas acerca del programa, son quienes asisten de manera regular a las sesiones, refieren que les brinda la oportunidad de aprender y sentirse escuchados. Asimismo, sostienen que ha favorecido a la adaptación a la vida universitaria, formulándose como un espacio de soporte emocional y orientación que les da la ocasión de expresar sus inquietudes con libertad.

Dentro de las fortalezas identificadas por los alumnos evaluados, se encuentran:

"Desarrollo de herramientas y técnicas que permiten avanzar / superarse, espacio para contar las dificultades / problemas, es motivador / levanta el ánimo."

"Las tutorías brindan soporte y favorecen el proceso de adaptación de los estudiantes de provincias. Lugar donde se pueden expresar con libertad."

Incluso, los estudiantes que abandonaron el programa, identifican fortalezas en el mismo:

"Los Programas/Las tutorías académicas son necesarias.

El Programa permitía organizarse (minoría). Brinda Orientación Vocacional."

Diversas investigaciones, demuestran que los estudiantes valoran las acciones directas o indirectas que implementan las instituciones para favorecer el proceso de adaptación de los alumnos, en el primer año académico. Asimismo, existe una mayor predilección por los docentes (tutores que tienden a fomentar relaciones basadas en la proximidad y apertura) (Amor, 2012; Haya, et al., 2013). 


\section{Debilidades del programa}

En definitiva, los estudiantes que renunciaron al programa tutorial presentan, en su mayoría, una percepción negativa del mismo. Considerándolo rutinario, invasivo, extenso (tiempo de sesión) y poco útil. Es importante señalar que gran parte de los alumnos que decidieron dejar el servicio se encuentran en ciclo superiores (II/III) y muestran un perfil diferenciado, ya que tienden a ser más autosuficientes e independientes, reservados en sus asuntos personales y, en algunos casos, algo herméticos. Otros reflejan ser cuestionadores y críticos e intolerantes.

"El tutorial no debería ser obligatorio, sino voluntario para las personas que lo necesiten, pero el académico sí, al menos 1 vez por semana."

(Estudiante que abandonó el Programa Tutorial - Sede Chorrillos)

"No ayudaba en el quehacer diario."

(Estudiante que abandonó el Programa Tutorial - Sede Surco)

"Brinda, más que nada, una ayuda emocional."

(Estudiante que abandonó el Programa Tutorial - Sede Surco)

"Las personas que van o creemos que deberían ir, son los que no se sienten seguros, los que se sienten tristes, inseguros o solos, los que están en riesgo."

(Estudiante que abandonó el Programa Tutorial - Sede Surco)

"Como me habían cambiado de tutor de nuevo, te preguntan todo porque no saben nada... es repetitivo."

(Estudiante que abandonó el Programa Tutorial - Sede Surco)
"Es un programa muy exploratorio y evaluativo. Preguntan mucho/ Preguntan temas personales. Es Rutinario."

(Estudiante que abandonó el Programa Tutorial - Sede Surco)

“Tener una sesión de una hora es muy extensa."

(Estudiante que permanece en el Programa Tutorial - Sede Chorrillos)

Un problema constante, que vienen a travesando las instituciones, es la falta de participación que tienen los alumnos universitarios dentro de los programas tutoriales; por ello, es importante que se sensibilice a los estudiantes acerca del real sentido de los mismos. Se debe fortalecer estos espacios, para que se conviertan en elementos fundamentales que influyan en el aprendizaje y la madurez de los pupilos; de esta manera, hacerles entender que el proceso formativo implica ir más allá del aprendizaje de contenidos (Álvarez Pérez y González Alfonso, 2007; Amor, 2012; Jiménez, 2010).

\section{Percepción del tutor}

Dentro de las fortalezas del tutor, la mayoría de participantes que acuden a las tutorías lo perciben en calidad de psicólogo, ya que cuenta con habilidades para escuchar al alumno cuando éste presenta problemas o dificultades; a la vez que el tutor, al ser una persona motivadora, brinda ánimo y soporte emocional, orientando y dando consejos para el mejor desempeño del pupilo.

Sin embargo, esta connotación de tutorpsicólogo incomoda a los estudiantes que ya no asisten a las tutorías, en la medida que pueden sentirse evaluados o que son jóvenes con problemas.

"Mi tutor me da ánimos, de todos los temas hablamos con mi tutor." 
(Estudiante que permanece en el Programa Tutorial - Sede Chorrillos)

“'El juego de la vida': Hablar con mi tutora es como que siempre me aconseja para seguir adelante como para ganar impulso en todo este proceso de la vida."

(Estudiante que permanece en el Programa Tutorial - Sede Chorrillos)

"La tutora te ayuda a desenvolverte mejor en el estudio, y a cómo mejorar mi tiempo, usar herramientas que te ayudan a mejorar el aprendizaje."

(Estudiante que abandonó el Programa Tutorial - Sede Chorrillos)

"Cuando estás decaído por dar un examen o cualquier cosa ella te da motivación personal."

(Estudiante que permanece en el Programa Tutorial - Sede Chorrillos)

"Si la verdad, si me ayudó a hablar sobre mis problemas, no era tan importante, pero me ayudaba a saber qué debo hacer y que no debo hacer."

(Estudiante que abandonó el Programa Tutorial - Sede Chorrillos)

"Yo si tuve una excelente experiencia con mi anterior tutora porque ella acomodaba sus horarios a mi disponibilidad. Por ejemplo, si nos faltaba una sesión ya me decía a qué hora estás disponible o de tal hora a tal hora estás disponible porque a veces tenía que recuperar horas de clase y le decía a tal hora no puedo, entonces de tal hora a tal hora yo puedo y coordinábamos."

(Estudiante que abandonó el Programa Tutorial - Sede Surco)

En resumen, las fortalezas de los tutores, identificadas por lo alumnos, son: habilidad para la escucha activa, receptividad ante los problemas y dificultades de los estudiantes, motivador/da ánimos para superarse, brinda soporte emocional, es orientador/da consejos, genera confianza para el diálogo, otorga herramientas para mejorar el rendimiento académico, brinda pautas para reforzar las habilidades sociales.

Es importante que los pupilos universitarios, en las diversas etapas de su desarrollo académico y profesional, aprendan a interpretar las diversas experiencias que atraviesan, consideren distintas perspectivas y encuentren un sentido a sus vidas. Por ello, los tutores deben asumir el reto y propiciar el empoderamiento de los estudiantes a través del fortalecimiento de sus capacidades de toma de decisiones, autorregulación, liderazgo, etc. (Adame Obrador, 2012; Castro, 2012; Lobato e Ilvento, 2013).

En cuanto a las debilidades de los tutores, las opiniones más críticas se encontraron en los alumnos que abandonaron las sesiones de tutoría. Estos participantes, perciben al tutor como una persona evaluadora respecto a la vida personal, deseando saber más allá de lo que quieren referir, tales como su estado emocional, problemas, familias, rendimiento, buscando que se desahoguen refiriendo sus dificultades. Esta exploración se hace reiterativa en las sesiones, tornándose aburridas, que hacen que el estudiante desista de acudir.

“Si, un poco más de confianza, pero no me gusta, es muy chibolo mi tutor, muy joven, como que una persona mayor como que se obtiene más experiencia, yo veo eso, muy difícil que un joven piense maduramente y no te pueda dar buenos consejos."

(Estudiante que permaneció en el Programa Tutorial - Chorrillos) 
"Yo pienso que deberían buscar temas interesantes como desenvolverse. Que hablen de más temas como habilidades sociales o liderazgo."

(Estudiante que permaneció en el Programa Tutorial - Chorrillos)

"Eso significa que la tutora mucho ahonda."

(Estudiante que abandonó el Programa Tutorial - Sede Chorrillos)

Por lo expuesto anteriormente, los alumnos (tanto los que continúan en el servicio tutorial, como los que abandonaron la tutoría) manifiestan expectativas respecto a un ideal de tutor. En ese sentido, los estudiantes señalan que los tutores deberían ser más dinámicos; activos; que propicien motivación en los demás; divertidos, ya que permitiría que las actividades se realicen de manera amena. Ser más empáticos, es decir, que sepan ponerse también en el lugar de los demás, ello genera más confianza y permite ser más amigos; que no se los perciba como psicólogos que están evaluando constantemente.

Es decir, el nuevo rol del profesor universitario implica asumir el reto de afrontar la diversidad del alumnado, acompañar al estudiante en su proceso de enseñanza aprendizaje y facilitar su desarrollo integral que le permita adquirir herramientas que lo prepare para la vida (Álvarez González, 2008; Amor, 2012).

Los tutores deben proponer temas innovadores y novedosos, de tal forma que generen curiosidad e interés en la realización de las actividades, y que cuenten con capacidad de poder ayudar a los jóvenes a organizarse en el desarrollo de sus hábitos de estudio.
"Deben enseñarnos como aprender más fácil, leer más rápido.”

"Técnicas más avanzadas".

(Estudiante que permanece en el Programa Tutorial - Sede Chorrillos)

"No tienen buena metodología, debería motivarnos para volverla más atractiva".

(Estudiante que abandonó el Programa Tutorial - Sede Surco)

"No debe ser aburrida, debe hacernos sentir en confianza, como un rompehielos."

(Estudiante que abandonó el Programa Tutorial - Sede Surco)

“Debe ser más dinámico, más lúdico."

(Estudiante que abandonó el Programa Tutorial - Sede Surco)

“Que sea más 'amigo y menos psicólogo', 'que juegue una pichanguita', 'que vayamos a almorzar con él'".

(Estudiante que abandonó el Programa Tutorial - Sede Surco)

"Que proponga nuevas cosas, que brinden talleres como Memoria rápida, cómo expresarse, cómo hablar / Oratoria."

(Estudiante que permanece en el Programa Tutorial - Sede Chorrillos)

Con respecto a la edad del tutor, no se percibe un consenso entre los participantes en la medida que algunos buscan:

- Una persona más madura, que cuente con mayor experiencia para poder orientarlos o aconsejarlos.

- Otros participantes consideran que debería ser más joven, que sea más cercano a sus intereses y sus estilos. 


\section{Evaluación de la permanencia en el programa tutorial}

Dentro de la principales razones para asistir y mantenerse en el programa tutorial, se encuentra la valoración y satisfacción con el programa, soporte emocional para la adaptación, vinculo positivo con el tutor, sentirse estresados y presionados (latente).

En cuanto a las razones para desertar, se encuentra la subvaloración del programa tutorial, considerado poco útil y reiterativo; percibirse como autosuficiente e independiente: "No lo necesito, eso es para las personas que están con problemas". Al ser muy reservados (casi herméticos en algunos casos), les desagrada que se indague mucho sobre sus cosas personales, además, la duración de las sesiones de tutorías es muy extensa.

Ante la pregunta de si es que ellos regresarían al programa tutorial, la mayoría manifiesta que «no» regresaría porque no es necesario o útil para ellos.

"Las tutorías deberían ser opcionales cuando una persona necesita sí, que vaya normal, que esté dispuesto el tutor a escuchar. Porque si no tienes nada que contar ya para qué vas a ir."

(Estudiante que abandonó el Programa Tutorial - Sede Surco)

"Yo creo que hay gente que lo necesita. Las personas que lo necesitan serían personas que tienen problemas, y la que no lo necesita no sé, yo tengo problemas pero yo los soluciono."

(Estudiante que abandonó el Programa Tutorial - Sede Chorrillos)

"No, porque te quita tiempo."

(Estudiante que abandonó el Programa Tutorial - Sede Surco)
El acompañamiento tutorial es sumamente importante en el proceso de adaptación de los estudiantes a la vida universitaria, pero éste debe responder a las necesidades y demandas académicas, socioemocionales, culturales y de desarrollo de los alumnos. Por ello, es necesario que se genere un espacio donde el tutor plantee estrategias prácticas y aplicables a situaciones cotidianas; que permitan un aprendizaje no sólo a nivel académico sino también que proporcionen herramientas aplicables a la vida.

El proceso de adaptación ha sido una experiencia difícil y complicada para gran parte de los participantes, por el desarraigo de su familia, de su hábitat y de sus costumbres. Estudiar en Lima ha implicado un cambio de vida y de asunción de nuevas responsabilidades no sólo educativas, sino también en su manutención personal que genera cuadros de ansiedad que el tutor debe ayudar a manejar. El programa tutorial resulta, en parte, ser un regulador emocional y un espacio donde el alumno puede expresar sus problemas y dificultades.

En ese sentido, los estudiantes reconocen que la tutoría favorece a la adaptación y contribuye con el proceso de asimilar la nueva vida que tienen y las exigencias que ésta implica, les brinda orientación y consejería en temas emocionales, sociales y en algunos casos de refuerzo académico, favorece la libertad para expresarse con libertad y sin temor; finalmente, es un medio que los ayuda a superarse y manejar las dificultades que se les presente.

A pesar de ello, es importante replantear la propuesta e incidir en el estilo de abordaje que emplea el tutor, ya que éste es identificado como invasivo y evaluativo debido a que profundiza demasiado en los temas personales. Se debe enfatizar en 
la diferenciación de un acompañamiento terapéutico y uno tutorial.

Por esta razón, se propone implementar una metodología que involucre un sistema lúdico y dinámico, de preferencia que el trabajo sea grupal, y que la temática sea planteada en función a un diagnóstico participativo.

Un aspecto importante, sería monitorear la percepción de los estudiantes mediante encuestas cortas que permitan realizar los ajustes necesarios con respecto al tutor, su mecánica de trabajo y los temas que se abordan.

\section{REFERENCIAS}

Adame Obrador, M. T. (2012). La orientación y tutoría en la universidad. Análisis de las realidades existentes. Educación y Cultura, 15, 215-232.

Álvarez González, M. (2004). Los modelos de orientación en España: presente y futuro. Revista Española de Orientación y Psicopedagogía, 15(2), 147-162.

Álvarez González, M. (2008). La tutoría académica en el espacio europeo de la educación superior. Revista Interuniversitaria de Formación del Profesorado, 22(1), 71-88.

Álvarez Pérez, P. y González Alfonso, M. (2007). El asesoramiento y la tutoría de carrera en la enseñanza superior: resultados de un programa de atención al alumnado de la Universidad de la Laguna. Revista Internacional de Investigación e Innovación Educativa, 9, 95-110.

Álvarez Pérez, P. y Jiménez, H. (Comp.). (2003). Tutoría universitaria. Tenerife: Servicio de publicaciones de la ULL.

Álvarez Pérez, P., Alegre de la Rosa, O. y LópezAguilar, D. (2012). Las dificultades de adaptación a la enseñanza universitaria de los estudiantes con discapacidad: un análisis desde un enfoque de orientación inclusiva. RELIEVE, 18(2). doi: 10.7203/ relieve.18.2.1986

Álvarez Rojo, V., García, E., Gil, J. y Romero, S. (2000). Orientación en los procesos de enseñanza-aprendizaje en la universidad. En H. Salmerón Pérez y V.L. López Palomo (Coords.), Orientación educativa en las universidades (pp. 47-67). Sevilla: Grupo Editorial Universitario.

Amor, M. I. (2012). La orientación y la tutoría universitaria como elementos para la calidad e innovación en la educación superior. Modelo de acción tutorial (Tesis doctoral). Universidad de Córdoba, España.

Apodaca, P. y Lobato, C. (1997). Calidad en la universidad: orientación y evaluación. Barcelona: Laertes.

Bausela, E. (2006). Áreas, contextos y modelos de orientación en intervención psicopedagógica. Revista Electrónica Diálogos Educativos, 12, 16-28.

Bishop, J. B. (1990). The university counseling center: An agende for the 1990s. Journal of Counseling and Development, 68, 408-413.

Bisquerra, R. (1998). Modelos de orientación e intervención psicopedagógica. Barcelona: Praxis.

Castellano, F. (1995). La orientación educativa en la Universidad de Granada. Granada: Servicio de Publicaciones de la Universidad de Granada.

Castellano, F. y Sanz, R. (1990). Un análisis de necesidades entre estudiantes de la Universidad de Granada para la elaboración de un programa de orientación educativa. Revista de Investigación Educativa, 8(16), 149 -155. Castro, R. (2012). Manual de tutoría y plan de acción tutorial basado en competencias 
profesionales. Chihuahua, México: Universidad Tecnológica de Chihuahua.

Colvin, J. W. (2007). Peer Tutoring and Social Dynamics in Higher Education. Mentoring \& Tutoring: Partnership in Learning, 15(2), 165-181.

Coriat, M. (Ed.) (2002). Jornadas sobre tutorías y orientación. Granada: Universidad de Granada.

Coriat, M. y Sanz, R. (2005). Orientación y tutoría en la Universitaria de Granada. Granada: Universidad de Granada.

Creswell, J., Hanson, W., Clark, V. y Morales, A. (2007). Qualitative research designs: Selection and implementation. The Counseling Psychologist, 35(2), 236264.

Díaz, G., Tarragona, M. y Trejo, N. (2007). Desarraigo cultural en adultos jóvenes profesionistas que residen en el extranjero. Episteme, 11(3).

Echeverría, B. (1997). Los servicios universitarios de orientación. En P. Apodaca y C. Lobato (Eds), Calidad en la universidad: orientación y evaluación (pp. 112-136). Barcelona: Laertes.

Ferrer, J. (2003). La acción tutorial en la universidad. En F. Michavila y J. García (Coords.), La tutoría y los nuevos modos de aprendizaje en la universidad (pp. 6784). Madrid: Consejería de Educación, Cátedra Unesco de la Universidad Politécnica.

Gairín, J., Feixas, M., Guillamón, C. y Quinquer, D. (2004). La tutoría académica en el escenario europeo de la educación superior. Revista Interuniversitaria de Formación de Profesorado, 18(1), 61-77.

García Nieto, N. (2008). La función tutorial de la universidad en el actual contexto de la educación superior. Revista Interuniversitaria de Formación del Profesorado, 22(1), 21-48.
García, J. M. y Gálvez, M. (1996). Un modelo tutorial universitario. Revista Complutense de Educación, 7, 51-66.

González López, I. y Martín Izard, J. F. (2004). La orientación profesional en la universidad, un factor de calidad según los alumnos. Revista Española de Orientación y Psicopedagogía, REOP, 15(2), 299-315.

Haya, I., Calvo, A. y Rodríguez-Hoyos, C. (2013). La dimensión personal de la tutoría universitaria. Una investigación cualitativa en la Universidad de Cantabria (España). Revista Española de Orientación y Psicopedagogía, 24(3), 98-113.

Jiménez, J. (2010). Hacia un nuevo proyecto de tutoría universitaria en el EEES. Revista Española de Orientación y Psicopedagogía, REOP. 21(1), 37-44.

Lázaro, A. (1997). La función tutorial de la acción docente universitaria. Revista Complutense de Educación, 8(1 y 2), 234252 y 109-127.

Lobato, C. y Echevarría, B. (2004). Tutorías y sistemas de apoyo a los estudiantes. En AA.VV., Pedagogía universitaria: hacia un espacio de aprendizaje compartido, Volumen 1, (pp. 299-301). Bilbao: Editorial ICE de la Universidad de Deusto y Ediciones Mensajero.

Lobato, C. e Ilvento, M. C. (2013). La orientación y tutoría universitaria: una aproximación actual. Revista de Docencia Universitaria. REDU. 11(2), 17-25.

Marín, M. A. y Rodríguez Espinar, S. (2001). Prospectiva del diagnóstico y de la orientación. Revista de Investigación Educativa, 19(2), 315-362.

Michavila, F. y García, J. (Eds). (2003). La tutoría $y$ los nuevos modos de aprendizaje en la universidad. Madrid: Consejería de Educación de la CM. 
Pantoja, A. y Campoy, T. (Coords.) (2009). Planes de acción tutorial en la universidad. Jaén: Servicio de Publicaciones de la Universidad de Jaén.

Pino, M. R. y Ricoy, M. C. (2006). La tutoría como recurso en la enseñanza universitaria. Estudio de necesidades y contribuciones actuales. Bordón, 58(3), 345-358.

Repetto, E. (2002). Modelos de orientación e intervención psicopedagógica. Volumen I. Marco conceptual y metodológico. Madrid: UNED.

Rodríguez Espinar, S. (Coord.). (2004). Manual de tutoría universitaria. Recursos para la acción. Barcelona: Octaedro- ICE U. de Barcelona.

Rodríguez Moreno, M. L. (2002). Hacia una nueva orientación universitaria. Barcelona: Ediciones de la Universidad de Barcelona.

Romo, A. (2011). La tutoría. Una estrategia innovadora en el marco de los programas de atención a estudiantes. México D.F.: Anuies.

Rué, J. (2004). La convergencia europea: entre decir e intentar hacer. Revista Interuniversitaria del Formación del Profesorado, 18(1), 39-59.

Saíz, C. M. y Roman, J. M. (2011). Cuatro formas de evaluación en educación superior gestionadas desde la tutoría. Revista de Psicodidáctica, 16(1), 141-161.

Sanz Oro, R. (2001). Orientación psicopedagógica y calidad educativa. Madrid: Pirámide.

Stone, G. L., y Archer, J. (1990). College and university counselling center in the 1990s: Challenges and limits. The Counseling Psychologist, 18, 539-607.
Upcraft, M. L., Gardner, J. N. y Barefoot, B. O. (Eds.) (2005). Challenging and supporting the first year student: a handbook for improving the first year of college. San Francisco: Jossey- Bass.

Watts, A. G. y Van Esbroeck, R. (1998). New skills for new futures. Higher Education Guidance and Counselling Services in the European Union. Bruselas: Fedora/ Vubpress.

Zabalza, M. A. (2003). Competencias docentes del profesorado universitario. Calidad y desarrollo profesional. Madrid: Narcea.

\footnotetext{
(C) Los autores. Este artículo es publicado por la Revista Digital de Investigación en Docencia Universitaria del Área de Investigación de la Dirección de Calidad Educativa, Universidad Peruana de Ciencias Aplicadas. Este es un artículo de acceso abierto, distribuido bajo los términos dela Licencia Creative Commons Atribución-CompartirIgual4.0 Internacional.(http://creativecommons.org/licenses/by-sa/4.0/), que permite el uso no comercial, distribución y reproducción en cualquier medio, siempre que la obra original sea debidamente citada.
} 